\title{
Kidney Changes After Daily Slow-bolus IV Injection of Polyoxyl 35 Castor Oil/Ethanol in 5\% Dextrose for 2 Weeks to Wistar Rats
}

\author{
K. Killary,* D. Diaz,* G. Argentieri,* R. Dugyala*, and K. Bowenkamp* \\ * Novartis Institutes for BioMedical Research, Translational Sciences, Preclinical Safety, \\ Investigative Pathology, Pathology, and Toxicology, One Health Plaza, East Hanover, NJ 07936
}

\begin{abstract}
Polyoxyl 35 castor oil USP (a.k.a. Polyoxyethylenglyceroltriricioleat 35 (DAG); Cremophor EL () is a non-ionic solubilizer and emulsifier used for aqueous preparations of hydrophobic substances in pharmaceuticals, cosmetics and feedstuffs. Polyoxyl 35 castor oil is soluble in water and ethanol, and the degree of solubilization achieved depends on the amount of Polyoxyl 35 castor oil used. Oral, topical, and inhaled delivery of Polyoxyl 35 castor oil is well tolerated in many species. Percutaneous administration of Polyoxyl 35 castor oil also is reported to be well tolerated in rats but may cause anaphylactoid reactions in cattle and humans. In a recent follow-up toxicity study, sterile-filtered 95\% Polyoxyl 35 castor oil/ethanol $(65 / 35 \mathrm{v} / \mathrm{v})$ in $5 \%$ Dextrose at a 1:10 ratio was used as the vehicle for once daily intravenous injection into the tail vein of Wistar rats for 2 weeks at volumes of 2 or $10 \mathrm{~mL} / \mathrm{kg} \mathrm{BW}$. Rats of both genders that received $10 \mathrm{~mL} / \mathrm{kg}$ Polyoxyl 35 castor oil/ethanol were pale and ataxic with decreased locomotor activity, and in males body weight gain and food consumption were decreased. No clinical pathology findings were attributable to the kidney. At necropsy paired kidney weight was increased in rats that received $10 \mathrm{~mL} / \mathrm{kg}$ Polyoxyl 35 castor oil/ethanol vehicle, and segmental vacuolation of proximal tubular epithelium occurred with higher incidence and severity. Vacuole contents were not labeled by Oil-Red-O staining for neutral triglycerides or lipids. Ultrastructural examination of vacuolated cells revealed increased numbers of distended lysosomes containing variably electron-dense granular material. Renal tubular vacuolation seen in this study was considered secondary to lysosomal accumulation of Polyoxyl 35 castor oil/ethanol vehicle or metabolites when injected by slow IV bolus daily to young-adult Wistar rats. ${ }^{\circledR}$ Cremophor EL is a registered trademark of BASF Aktiengesellschaft.
\end{abstract}

Materials and Methods: Animals: IGS Wistar Hannover Rats (CRL:WI(HAN)) approx. 10 weeks old and 175 - $400 \mathrm{~g}$ body weight (BW) at study start. Test articles: Polyoxyl 35 castor oil/ethanol $(65: 35 \mathrm{v} / \mathrm{v}) 95 \%$ diluted with $5 \%$ Dextrose injection USP at a 1:10 ratio of Polyoxyl 35 castor oil/ethanol was filtered using $0.22 \mu \mathrm{m}$ Durapore (PVDF) membrane and administered by intravenous (slow-bolus) injection into the tail vein once daily for 2 weeks at dose volumes of either 2 or 10 $\mathrm{mL} / \mathrm{kg} \mathrm{BW}$. A 5\% dextrose solution (5\% Dextrose Injection, USP) was administered by once daily slow-bolus IV injection for comparison. Standard toxicology study endpoints were collected.

TEM: Archived NBF-fixed kidney was post-fixed and processed by microwave method (Modified Karnovsky's fixative and 1\% osmium tetroxide in cacodylate buffer, dehydrated through a graded water/acetone series, infiltrated and embedded in epoxy resin (EMBed 812, Electron Microscopy Sciences, Hatfield, PA). Toluidine blue-stained $0.6 \mu \mathrm{m}$ sections were screened to prepare uranyl acetate \& lead citrate-stained, 600-700 A sections mounted on 200 mesh copper/rhodium grids for TEM and digital photomicrography ( $\geq 4$ images) with an Olympus Soft Imaging System Morada digital camera mounted on an FEI Tecnai G2 BioTwin transmission electron microscope. 
Conclusions: Once daily slow-bolus IV injection of $10 \mathrm{~mL} / \mathrm{kg}$ Polyoxyl 35 castor oil/ ethanol $(65: 35 \mathrm{v} / \mathrm{v}) 95 \%$ diluted with 5\% Dextrose injection USP to rats for 2 weeks causes several changes:

- Pallor, decreased locomotor activity, ataxia, body weight and body weight gain in both sexes; decreased food consumption in males

- Increased mean relative kidney weight vs. body weight in both sexes and vs. brain weight in males

- Segmental proximal tubular epithelial vacuolation in the kidney of both sexes unassociated with accumulation of neutral triglycerides or lipids or altered serum/urine chemistry

- In males, increased proximal tubular accumulation of globular to crystalline material (presumptive alpha $2 \mu$-globulin)

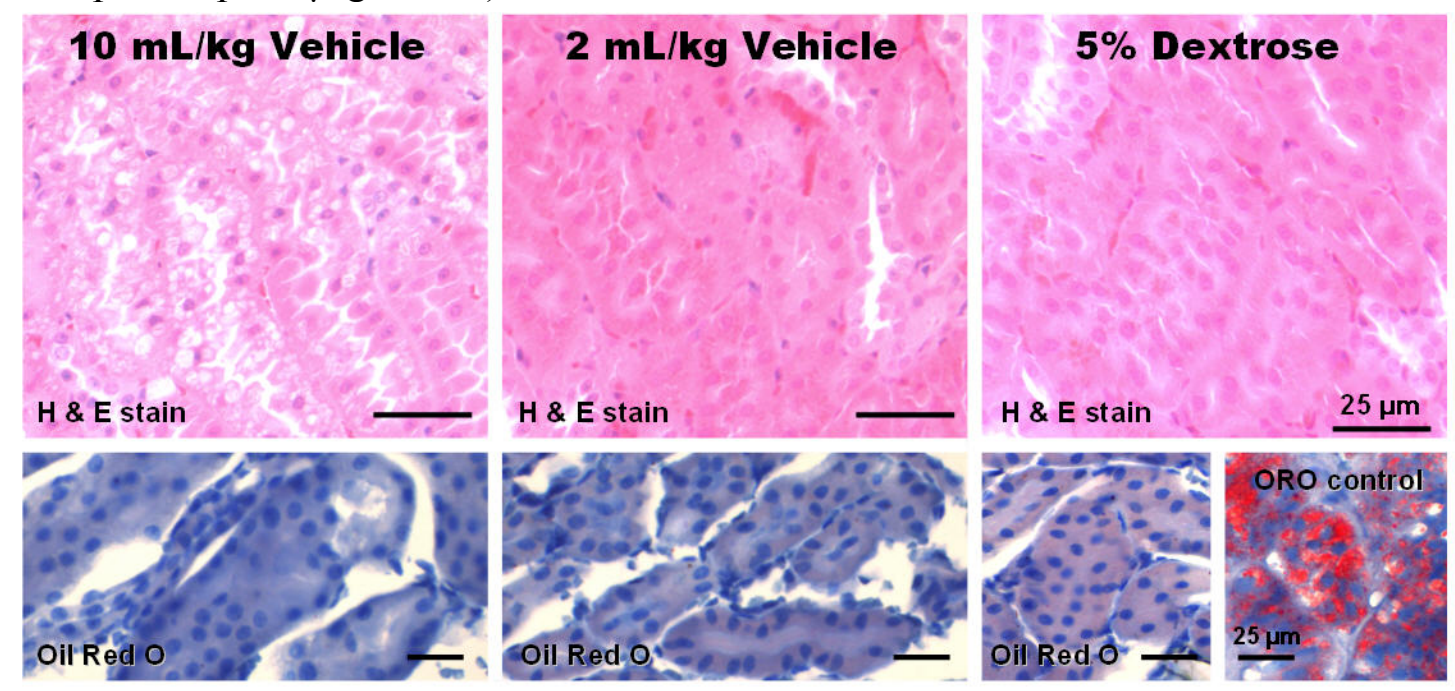

FIG. 1. Histopathology and Oil Red O staining: Polyoxyl 35 castor oil/ethanol vehicle administered at $10 \mathrm{~mL} / \mathrm{kg}$ to rats caused segmental proximal renal tubular epithelial vacuolation without lipid or neutral triglyceride accumulation.
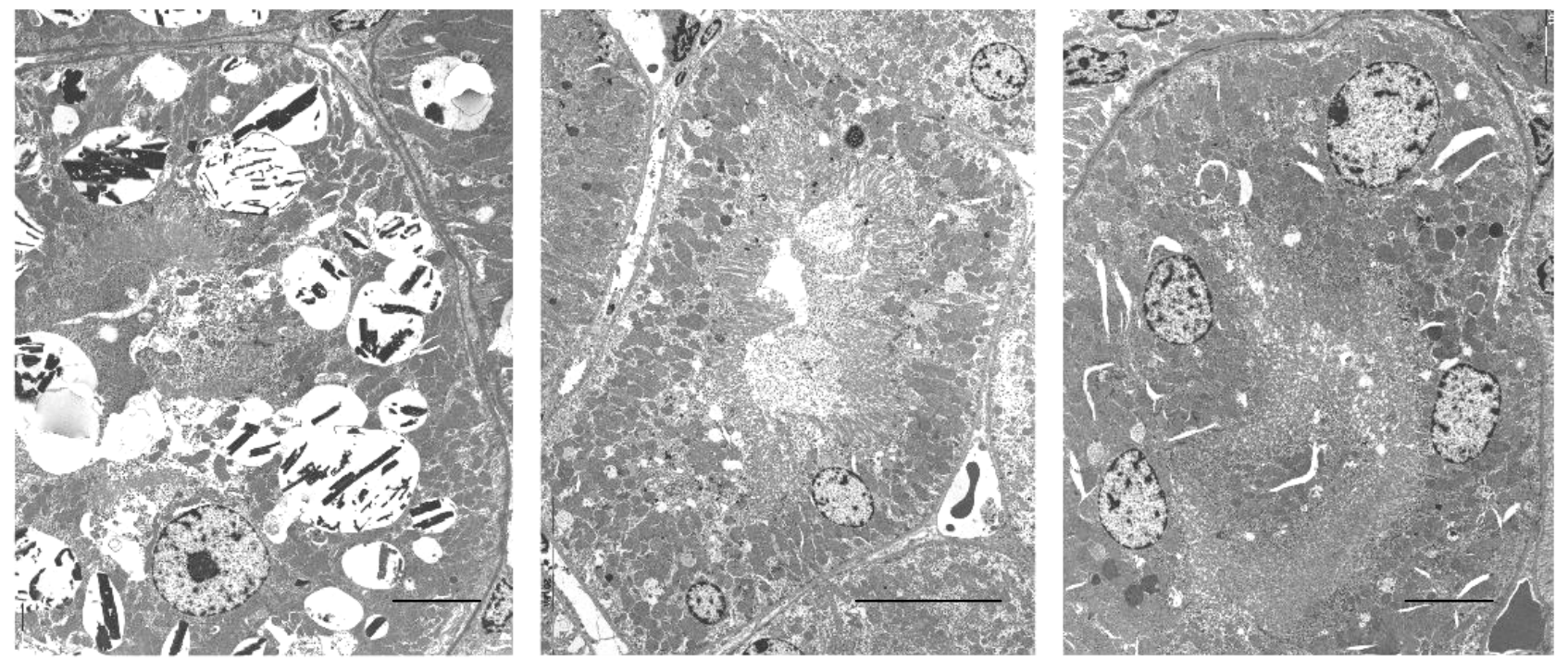

FIG. 2. Transmission Electron Microscopy: A. $10 \mathrm{~mL} / \mathrm{kg}$ vehicle. B. $2 \mathrm{~mL} / \mathrm{kg}$ vehicle. C. $5 \%$ dextrose. Proximal renal tubular epithelium of $10 \mathrm{~mL} / \mathrm{kg}$ Polyoxyl 35 castor oil/ethanol - treated rats contained many enlarged lysosomes filled with acicular crystalline material. Bar equals approximately $15 \mu \mathrm{m}$. 